\title{
Genealogía de una vejez no anunciada: biopolítica de los cuerpos envejecidos o del advenimiento
} de la gerontogubernamentalidad

\author{
Mario Ociel Moya
}

Programa de Doctorado en Antropología. Instituto de Investigaciones Arqueológicas y Museo R.P. Gustavo Le Paige S.J. Universidad Católica del Norte. San Pedro de Atacama, Chile. Becario CONICYT.

Email: mmoya@ucn.cl

Resumen: Lejos de aquellas descripciones emanadas de las ciencias sociales que muestran al envejecimiento y vejez de la población como un fenómeno sin historia y apolítico, el presente artículo pretende mostrar cómo el proceso de envejecimiento de la población obedece a prácticas de cálculo, gestión y control de la población iniciadas con la Constitución Política de 1925 en Chile. Hoy, la población de 60 y más años, forma parte de las actuales técnicas de gobierno que buscan la seguridad social y económica bajo una racionalidad biopolítica de la salud del cuerpo envejecido. ${ }^{1}$ salud pública.

Palabras clave: Vejez, biopolítica, gerontogubernamentalidad, cuerpo,

\section{Genealogy of an unannounced old age: biopolitics of aging bodies or the advent of gerontogovernmentality}

Abstract: Far from those descriptions emanating from the social sciences that show aging of the population as a phenomenon without history and apolitical, this article shows how the process of aging is due to accounting practices, management and population control that started with the 1925 Political Constitution in Chile. Today, people aged 60 and over is a target of governmental policies aiming toward social and economic security, under a biopolitical rationality towards the health of an aged body. health.

Key words: Old age, biopolitics, gerontogovernmentality, body, public

\section{Genealogia de uma velhice sem aviso prévio: biopolítica dos corpos de envelhecimento ou o advento da gerontogubernamentalidad}

Resumo: Longe de essas descrições que emanam das ciências sociais que mostram o envelhecimento e envelhecimento da população como um fenômeno sem história e apolítico, este artigo mostra como o processo de envelhecimento é 
devido a práticas contábeis, gestão e controle da população iniciada com a Constituição de 1925, no Chile. Hoje, a população com 60 anos ou mais de idade, faz parte das técnicas atuais de governo que buscam a segurança social e econômica sob uma racionalidade biopolítica da saúde do envelhecimento do corpo. saúde do corpo.

Palavras-chave: Envelhecimento, biopolítica, gerontogubernamentalidad,

"No se dirá ni repetirá nunca lo suficiente hasta qué punto la ilusión de lo natural y la ilusión de que "siempre ha sido así” ( ) y la amnesia de la génesis en la que se enraíza, constituyen un obstáculo al conocimiento científico del mundo social”.

Pierre Bourdieu.

El Ministerio del Ministerio.

"La idea de una ciencia neutra es una ficción, y es una ficción interesada, que permite considerar científica una visión neutralizada y eufemística (y por lo tanto particularmente eficaz simbólicamente porque es particularmente desconocible) de la representación dominante del mundo social”

Pierre Bourdieu.

Los usos sociales de la ciencia.

Las actuales descripciones del envejecimiento y la vejez en Chile, hoy tan de moda en algunos espacios académicos y político-discursivos, presentan este fenómeno con ciertas características que nos proponemos problematizar. Por un lado, la vejez despojada de historia ${ }^{2}$, cuyo proceso de producción parece ser olvidado, es mostrada como un fenómeno novedoso en la humanidad y propio de las sociedades modernas, un fenómeno colmado de desafíos actuales y futuros que nos parecen ciertos y atinado plantearlos, sin embargo, bastante lejos de un componente político que en su conformación, actualidad y futuro, ha jugado, juega y jugará un papel fundamental en su gestión y control.

Para la observación de nuestro problema proponemos considerar la articulación contenida en el concepto de biopolítica; andamiaje conceptual que alude a la observación y problematización de las lógicas de poder que consideran la vida, su estabilidad y manejo, al menos en dos niveles, a saber: a) nivel individual mediante la salud del cuerpo (el gobierno de sí), y b) mediante la salud de la población (el gobierno de los otros).

Así, sostendremos la tesis que la vejez es una construcción eminentemente biopolítica y técnico-científica, que emerge a partir del proyecto modernizador iniciado en la década de 1920 en Chile. Las consecuencias actuales de este fenómeno, emanadas del campo académico y de algunas agencias internacionales, ofrecen descripciones de inestabilidad respecto 
de lo social y, particularmente, respecto de lo económico. Asimismo, el devenir de la gestión y tratamiento de la vejez, tendría en la actualidad por parte del Estado, mecanismos homólogos a los aplicados a las llamadas minorías, materializando en sus prácticas el binomio inclusión/exclusión como parte de una racionalidad biopolítica.

\section{Algunas consideraciones sobre [Bio]política}

"Me parece que uno de los fenómenos fundamentales del siglo XIX fue y es lo que podríamos llamar la consideración de la vida por parte del poder; por decirlo de algún modo, un ejercicio del poder sobre el hombre en cuanto ser viviente, una especie de estatización de lo biológico o, al menos, cierta tendencia conducente a lo que podría denominarse la estatización de lo biológico".

Michel Foucault.

Defender la sociedad.

Limitaremos aquí el uso del concepto biopolítica a la cuestión de la salud y la población, pues analizar sus reales alcances no es el propósito en este texto ${ }^{3}$. Proponemos, entonces, enunciar algunas de las particularidades de este concepto popularizado en la década del 70 por Foucault (2002a, 2002b, 2007, 2008) en dirección a nuestro problema y su posterior análisis.

En La voluntad de saber (2002a), Foucault propone fijar el desarrollo del interés político por la vida en el siglo XVII bajo dos formas específicas: la primera, centrada en el cuerpo como máquina (anátomo-política), que se materializa con el sometimiento y corrección del cuerpo o del sujeto desviado (sociedad disciplinaria); y la segunda, desde mediados del siglo XVIII, cuyo objetivo es el cuerpo-especie (biopolítica de la población), centrada en la generación de medidas de intervención y gestión sobre las tasas de natalidad, el manejo del binomio salud/enfermedad y la postergación de la muerte. De esta forma, () las disciplinas del cuerpo y las regulaciones de la población constituyen los dos polos alrededor de los cuales se desarrolló la organización del poder sobre la vida (Foucault, 2002a:168169), polos que no funcionarían de manera excluyente, sino por el contrario, de forma complementaria.

Fundamentales, entonces, son los conceptos de seguridad y gubernamentalidad enunciados por Foucault en los cursos Seguridad, territorio y población (2007) y El Nacimiento de la biopolítica (2008), pues estos conceptos sedimentan el espacio para la producción de la biopolítica. El concepto de seguridad albergaría aquellas prácticas que forjan la reactivación y transformación de las técnicas jurídico-legales y disciplinarias que buscan mantener un orden social específico, en donde no sólo se desarrollan las viejas estructuras de la ley y la disciplina, sino también ciertos mecanismos sutiles que buscan erradicar o regular (por ejemplo, me- 
diante campañas médicas preventivas) aquellos aspectos que resultan problemáticos para la sociedad. Así, sobre el cuerpo social, se tornaría necesaria la aplicación de ciertos mecanismos que permitiesen que fenómenos como el envejecimiento se mantengan dentro de los límites () que sean social y económicamente aceptables y alrededor de una media que se considere, por decirlo de algún modo, óptima para un funcionamiento social dado (Foucault, 2007:20).

Para la mantención y reproducción de estas lógicas, la gubernamentalidad es otro elemento clave. Con este concepto Foucault intentó definir un tipo de poder específico, una racionalidad que se materializa y reproduce de manera atomizada, como la racionalidad inmanente a los micropoderes, cualquiera sea el nivel considerado (relación padres/hijos, individuo/poder público, población/medicina, etcétera) (Senellart, 2007:449). Entonces, en un doble juego, la gubernamentalidad es interior y exterior al Estado, ya que son precisamente las tácticas de gobierno las que definen su posición y formas de accionar.

Ahora bien, Foucault escribe y piensa desde Europa; sin embargo, considerado el envejecimiento como un devenir, un continuo socio-histórico, creemos plausible una lectura biopolítica de fenómenos como el proceso de envejecimiento y vejez experimentado en Chile, al evidenciar la perpetración de un proceso en donde el cuerpo y el discurso de protección de la vida se han apoderado de manera paulatina de las lógicas al interior del campo político. Un trabajo que a nuestro juicio refleja de manera notable dicho proceso, es el de Illanes (2007), quien muestra cómo el cuerpo, en especial el cuerpo empobrecido, comienza a ser intervenido entre $1887 \mathrm{y}$ 1940 por fundaciones y el Estado, con el objetivo de mantener y armonizar el sistema social frente a la creciente tensión producida por la diferencia de clases. Dicho proceso, según la autora, sigue las directrices de desarrollo y modernización del occidente europeo que definirían, además, los lineamientos de la política social del siglo XX en América Latina. En el caso chileno, la difusión de ideas, discursos y nuevas prácticas en salud, responde, según Zárate (2008), a la formación de médicos en Europa, quienes trajeron al país aquellas ideas modernizadoras que comienzan a gestarse a partir de la década del 20 como un ideario político. De esta forma, medicina, academia y política, comienzan a constituirse como los principales agentes, ideadores y ejecutores de prácticas en torno al cuerpo, que configurarán formas legítimas de intervención a nivel poblacional.

\section{El advenimiento de la vejez en Chile: ¿una consecuencia perversa del proyecto moderno?}

Preguntarse por el envejecimiento como una consecuencia perversa del proyecto moderno, es tensionar el fenómeno y posicionarlo como una nueva anomalía social. Anomalía, en tanto fenómeno a nivel demográfico cuyas consecuencias, dimensionadas esencialmente por los expertos de las 
diversas áreas del conocimiento, estarían asociadas a la inestabilidad social y a la merma económica de los Estados. Paradojalmente, dichas descripciones son homologas a las realizadas a partir de la década del 60 respecto del baby boom, fenómeno que, como mostraremos más adelante, se encuentra estrechamente relacionado con la actual situación de envejecimiento.

El envejecimiento de la población es el resultado de una serie de variables vinculadas a las mejoras sanitarias, cambios en el comportamiento reproductivo de las mujeres y políticas dirigidas al manejo y cuidado de la población. En efecto, el eco en Latinoamérica del tránsito de lo privado a la esfera pública, graficado principalmente en el rol activo del Estado mediante el desarrollo de la higiene pública que experimentó Europa a fines del siglo XVIII (Foucault, 2002), no se materializó en América Latina hasta fines del siglo XIX (Zárate, 2008). En el caso chileno, comienza el proceso con una sucesión de políticas formalizadas en la constitución de $1925^{4}$ que buscaban la disminución de las altas tasas de mortalidad producto de las enfermedades infecciosas y parasitarias que caracterizaban el perfil epidemiológico del país.

Mancomunadamente con la creciente profesionalización de la medicina, un amplio repertorio de instituciones y normativas dirigidas al bienestar higiénico son un testimonio de esta transición clave que experimentaba la salud () (Zárate, 2008:15). Así, la implementación de políticas sanitarias por parte del Estado, junto con otras iniciativas institucionales, particularmente, impulsadas por las escuelas de medicina, se inició un fuerte proceso de intervención a nivel poblacional, con políticas e iniciativas asociadas a diversos temas identificados como contingentes, tales como: el tratamiento del alcoholismo mediante la instauración de los primeros programas de salud mental localizados en los sectores empobrecidos del país; el manejo, prevención y tratamiento de enfermedades venéreas, con incipientes programas de educación sexual; la problemática del aborto y la planificación familiar ${ }^{5}$. Estas iniciativas irían paulatinamente interviniendo, reestructurando y en algunos casos, restringiendo formas de comportamiento y la relación que los sujetos tenían con su propio cuerpo. De esta manera el cuerpo físico del pueblo, su necesidad y derecho de vivir adquiere en el siglo XX () el carácter de una categoría ética, ideológica, política y económica (Illanes, 2007:15) reproduciendo, de esta manera, la racionalidad biopolítica europea.

Sin querer simplificar y reducir la complejidad de estos procesos, es necesario centrarnos en dos variables que presentaron una incidencia directa en los cambios demográficos experimentados en Chile y que despuntaron con el envejecimiento: el descenso de la mortalidad y el posterior control de la población en razón de su crecimiento. En efecto, como resultado del paulatino descenso de la mortalidad, tanto infantil como en adultos, la población comenzó a experimentar un aumento exponencial que encarnó la preocupación de algunos políticos y médicos que veían, con sospecha malthusiana, los posibles efectos negativos que tendría sobre el 
país. A saber, entre los años 1940 y 1960, la tasa bruta de mortalidad bajó de 21 a 13 defunciones promedio por cada mil habitantes. De manera paralela, la tasa bruta de natalidad aumentó de 36 a 38 nacimientos promedio por mil habitantes. Como consecuencia, el crecimiento natural de la población aumentó de 15 a 25 personas promedio por cada mil habitantes en ese período (Villalón, 2012). De esta forma, dicha preocupación, según los expertos nacionales y agencias extranjeras, se fundamentaba en dos cuestiones centrales: por un lado, en lo socioeconómico, por el alto costo asociado a la mantención de la población, y por otro, en lo sanitario, ya que el sistema no daba abasto a las nuevas necesidades de una población con alto nivel de crecimiento.

En base al aumento de los nacimientos a nivel país, comienzan a materializarse los programas de control familiar a partir de la década del 60. Este nuevo dispositivo para el control y manejo de la población, según Pieper (2008), responde también a la tendencia que la política exterior de los Estados Unidos comienza a diseminar en las regiones en desarrollo. En tal sentido, las fundaciones privadas como el Milbank Memorial Fund, la Rockefeller, la Ford Foundation y el Population Council, hicieron eco de la declaración del presidente estadounidense Lyndon Johnson, en 1965: menos de cinco dólares invertidos en el control de la población valen un centenar de dólares invertidos en crecimiento económico. (Pieper, 2008:206).

En esta misma línea, en Chile, fue el médico Benjamín Viel Vicuña ${ }^{6}$ quien mostró especial interés por el tema, interés que se materializa en una publicación en 1966 titulada La Explosión Demográfica: ¿Cuántos son demasiados?. En ella, Viel desarrolla el propósito de la planificación familiar y el vínculo que tendría con la modernización de la familia y del país. Por otro lado, en su práctica médica, Viel desarrolló trabajo en sectores empobrecidos de Santiago y en zonas rurales con el objeto de llevar las ventajas atribuidas a la planificación familiar a todas las mujeres sin que estuviese mediado por su situación socioeconómica ${ }^{7}$.

En este contexto, en la segunda mitad de la década del 60, comienza el declive en las tasas de fecundidad en los sectores más acomodados del país. A nivel país, en la década del 50 el promedio de hijos por mujer estaba sobre los 5, identificándose una primera fase de disminución acelerada hasta la segunda mitad de los años 80 que llegó a los 2,7 hijos y una segunda fase de descenso moderado de 2,4 hijos para el año 2000 por mujer en edad fértil. Según las proyecciones realizadas por CELADE e INE, indicaban que en el periodo 2000 - 2005 la tasa global de fecundidad sería de 2,4. Sin embargo, las estadísticas vitales señalan que la tasa en los últimos años es inferior a 2,0, por tanto, menor a la proyectada (Palma, 2006:2).

En la conjunción de la disminución de la tasa de mortalidad evidenciada desde la década del 20, junto con la disminución de la tasa de natalidad en los años 60 y el aumento en la esperanza de vida desde mediados del siglo XX (Expansiva, 2008), se identifica la principal causa que explica el aumento de personas en el grupo de edades entre los 15 y 59 años, y aque- 
llos con 60 y más años (segundo y tercer tramo de edad respectivamente). Ahora bien, no sólo en la modificación en estos grupos de edades se materializa dicho proceso, sino también en la disminución sostenida de la población ubicada en los 0 y 14 años de edad (primer tramo) lo que nos lleva en la actualidad a hablar de Chile como un país envejecido y en una etapa de transición avanzada ${ }^{8}$.

Ahora bien, en la búsqueda de reconstruir el devenir del envejecimiento como problemática, no debemos olvidar el rol que han tenido las agencias internacionales en esta labor. En tal sentido, situamos a la ONU como la organización que inicia un trabajo y enfoque analítico sobre el envejecimiento al comenzar sus estudios sobre el tema en 1948 (Terrón, 2004). Por otro lado, en el intento de avanzar en este posicionamiento, en 1977 la ONU plantea la necesidad de una Asamblea Mundial sobre Envejecimiento, decidiendo al año siguiente que dicha asamblea sea realizada en 1982. A partir de estos hechos, han sucedido una serie de eventos ${ }^{9}$ que han determinado lineamientos, resoluciones y declaraciones en torno a la población envejecida ${ }^{10}$, que los Estados han asumido y acomodado a sus contextos sociales específicos.

Más allá de la simple enunciación de los hitos que marcan el surgimiento del envejecimiento como problema, podemos concluir inicialmente lo siguiente: En primer lugar, el envejecimiento de la población es un resultado no previsto del proceso de modernización experimentado en el país, pues, como resulta obvio, las diversas intervenciones sobre la población no buscaban envejecerla, sino alcanzar estándares de salud colectiva acorde a los países europeos.

En segundo lugar, el rol de los expertos, en este caso médicos y salubristas, ha sido fundamental en la definición de prácticas asociadas al cuidado del cuerpo individual y social, acción que no ha estado, como vemos, al margen de ciertos componentes ideológicos y políticos. El trabajo mancomunado del Estado y las principales instituciones sociales a cargo de la salud, han legitimado socialmente las diversas intervenciones sobre la población bajo un discurso y práctica que traslada aspectos de la esfera privada a la pública. Así, la salud pública ${ }^{11}$, según creemos, materializa el área de conjunción entre ideología (política) y conocimiento (ciencia/técnica), pues es el espacio por excelencia desde donde es posible observar el gobierno de la vida y la reproducción de sus técnicas de poder y gestión.

En tercer lugar, estamos frente a dos hechos fundamentales que debemos considerar: primero, frente a una transición epidemiológica asociada a la disminución en las prevalencias, incidencias y mortalidad de las enfermedades infectocontagiosas, junto con el aumento en las incidencias, prevalencias y mortalidad de las enfermedades crónico degenerativas. Un segundo hecho, es el surgimiento de un nuevo segmento de la población, de una nueva etapa del ciclo vital que se intercala entre la jubilación y el deterioro que generan los estados avanzados de envejecimiento ${ }^{12}$ (Osorio, 2006c). Como veremos, este nuevo segmento se torna campo fértil para lo 
político, lo que se grafica en la cada vez mayor presencia en discursos, en las políticas sociales y en el número creciente de instituciones vinculadas a la vejez.

\section{Institucionalización del envejecimiento}

"Estamos alargando la esperanza de vida de los chilenos y si estamos alargando la esperanza de vida, tendremos que ser capaces también de alargar la calidad de vida de esos adultos mayores. Llegar a adulto mayor es comenzar una nueva etapa en la vida () Los países del futuro se van a caracterizar por la forma de cómo son capaces de organizar y aprovechar el mayor tiempo que tiene el adulto mayor ()".

Discurso del ex presidente Ricardo Lagos en el encuentro Vivir la vida, toda la vida, 2005

Producida la población envejecida, se instala la necesidad de crear organismos estatales y privados dedicados al abordaje de temas relativos a la salud, bienestar y seguridad social. Si bien la institución del Estado más representativa en este tema es el actual Servicio Nacional del Adulto Mayor (en adelante SENAMA), el origen de la producción de instituciones especialistas se sitúa en la década del $70^{13}$.

Para Morales (2010), si bien en el gobierno militar no existió una política de Estado en torno a la vejez, sí hubo líneas de trabajo y gestión dirigidas a esta población como resultado de las políticas asistenciales y de seguridad social en el marco del modelo económico neoliberal que comienza a implementarse. Por ejemplo, en el bienio 1980 y 1981, el gobierno militar pone en manos de privados la seguridad social, quienes se constituyeron como administradoras de Fondos de Pensiones (AFP), destinadas a tutelar el ahorro de los trabajadores para el momento del retiro (Morales, 2001:80). Eso sí, deja a la población de menos recursos bajo el alero estatal en el Instituto de Normalización Previsional INP ${ }^{14}$. Conjuntamente con esto, se desarrollaron otras instancias institucionales de carácter asistencial que trastocaron directamente a la población envejecida más vulnerable, como fue la creación de la Pensión Asistencial - PASIS (focalizado en los envejecidos en situación de pobreza) y la formación de la Comisión Nacional para la Protección de la Ancianidad - CONAPRAN en el periodo de 1974 y $1989^{15}$. En este sentido, la administración de las prácticas cotidianas de la población de 60 y más años, es la constante que identificamos desde la creación de CONAPRAN, cuyas intervenciones de carácter asistencialista, realizadas bajo la lógica del voluntariado, iban desde la conformación de clubes ${ }^{16}$ para personas mayores y hogares de ancianos [sic].

A partir de la vuelta a la democracia, bajo el primer gobierno de la Concertación a principios de los 90, Patricio Aylwin identifica a la población de 60 años y más como un sector prioritario para la política pública. 
No obstante, en esta primera etapa de institucionalización formal de la temática, sólo se elaboraron diagnósticos que describían el escenario social y económico de la población envejecida, para crear luego, en 1995 con Eduardo Frei Ruiz-Tagle en la primera magistratura, la Comisión Nacional para el Adulto Mayor. Un año más tarde, en 1996, el Consejo de Gabinete de Ministros del Área Social aprueba la Política de Gobierno sobre el Adulto Mayor. Por otro lado, en un intento de descentralizar la ejecución de las políticas, en 1997 se crean los Comités Regionales para el Adulto Mayor, cuyas principales funciones fueron la de administrar los fondos en regiones, la de asesorar al Intendente Regional en los planes y programas específicos y, naturalmente, la de implementar la política nacional para el adulto mayor.

En 1999, en el marco del Año Internacional del Adulto Mayor, el Gobierno de Chile envía al Congreso el Proyecto de Ley que crea el Servicio Nacional del Adulto Mayor - SENAMA, que se promulga bajo el gobierno de Ricardo Lagos ${ }^{17}$, cuyos principales objetivos fueron la promoción de los derechos (bajo una lógica de intervenciones de carácter participativo y fondos concursables que apuntan a la organización y productividad de esta población) y la elaboración de un sistema de protección social, centrado básicamente en las reformas al sistema de pensiones que en la actualidad se han implementado en el país ${ }^{18}$.

Estas instituciones y sus variantes actuales, como ya podrá suponer el lector, han cumplido, y cumplen, roles específicos en el manejo de esta población, gubernamentalizando saberes y prácticas asociados principalmente a la participación social y salud. Esta última dimensión, según creemos, se ha acentuado en la actualidad con aquellas lógicas de auto-cuidado y envejecimiento exitoso o activo $^{19}$ que son difundidos por instituciones académicas y el Estado.

En esta dirección, y considerando los antecedentes antes descritos, la población envejecida comienza a tener un tratamiento homólogo a las llamadas minorías en al menos dos sentidos: el primero de ellos, mediante la creación de instituciones especialistas en su tratamiento y gestión mediante la aplicación de saberes técnicos y específicos que fragmentan el cuerpo social en etnias, niños o viejos; y, el segundo, mediante la divulgación de lógicas participativas muy similares a las mostradas por Boccara (2007) y Boccara y Bolados (2008), respecto de la situación de la población atacameña en la segunda región del país.

\section{Algunos condicionamientos actuales: empoderamiento, participación y gobierno de sí como nuevo arte de gobierno}

“Ha habido, en el curso de la edad clásica, todo un descubrimiento del cuerpo como objeto y blanco de poder. Podrían encontrarse fácilmente 
signos de esta gran atención dedicada entonces al cuerpo, al cuerpo que se manipula, al que se da forma, que se educa, que obedece, que responde, que se vuelve hábil o cuyas fuerzas se multiplican”.

Michel Foucault. Vigilar y Castigar.

Hemos decidido tomar como muestras las ideas del empoderamiento y la participación social del adulto mayor, para observar, de manera somera, el tratamiento del envejecimiento y la vejez en la actualidad, pues estas nociones conforman los pilares fundamentales, según hemos observado, en su gestión y cálculo.

En términos precisos, como política de vejez, se han creado instancias centradas en la participación: la primera de ellas son los denominados Consejos Asesores Regionales de Mayores que, según lo explicitado por el propio SENAMA, es una iniciativa que surge para dar respuesta concreta a la necesidad de fortalecer la participación social y el ejercicio de la ciudadanía activa de las personas mayores que participan en las organizaciones sociales (SENAMA, 2009:105). Estos consejos ${ }^{20}$, que surgieron como una instancia de asociación y participación regional, fueron formalizados por el decreto presidencial 008/2008, asumiendo la misión de asesorar, apoyar e incidir en el proceso de diseño y ejecución de proyectos y programas dirigidos a la población envejecida, pues, hasta su formalización, estas instancias sólo tenían el carácter de iniciativas comunales no contando con representatividad regional.

Otra iniciativa impulsada por el SENAMA dirigida a la participación, es el Programa de Formación Continua para Dirigentes Mayores, que tiene por fin empoderar a los Consejos Regionales de Mayores, como a las diversas redes organizativas existentes a nivel regional. En este marco, mediante la figura de la Escuela de Dirigentes, se busca específicamente promover la satisfacción de las necesidades básicas de la población envejecida, su inclusión en la sociedad, su desarrollo, como también, promover, la (auto)protección de su salud. Finalmente, también en este escenario, surgen las Jornadas por el Derecho a Saber, cuyos principales objetivos son informar y dar cuenta de los avances de las políticas y programas que SENAMA ejecuta; establecer los puentes de comunicación necesarios con los grupos de adultos mayores organizados a nivel regional y local, y lograr, por parte de la población, una fiscalización a las diversas iniciativas asociadas a la vejez que el Estado diseña y ejecuta.

De lo expuesto por SENAMA, surgen interrogantes respecto a la idea que, para el fortalecimiento de la ciudadanía, condición sine qua non es la participación en las instancias organizadas de trabajo y gestión (consejos regionales, uniones comunales, juntas de vecinos, clubes de adultos mayores, centros culturales, etc.):

A ver, para postular a fondos concursables, como el Fondo Nacional 
del Adulto Mayor, es un requisito estar organizado, así que ahí hay también una intención de que los viejos se organicen () es como un fin utilitario de fondo, la idea es que se organicen () porque los viejos así como atomizados no pueden llevar adelante sus demandas () (Entrevista a funcionario de la Unidad de Estudios, SENAMA) ${ }^{21}$

En efecto, desde esta perspectiva, la organización social es lo que ayudaría a satisfacer necesidades y, lo principal, comunicarlas, ya que el reclamo del viejo de manera individual no tendría el efecto sobre las instituciones de Estado. Desde esa lógica, según creemos, se produce y reproduce el binomio inclusión/exclusión, en donde instancias que intentan incluir a un segmento de la población, a su vez, lo excluyen ${ }^{22}$ por no formar parte de los conglomerados institucionalizados por el Estado para una participación direccionada y operativa.

En este sentido, según Alguacil, la participación, en consecuencia, es el nexo que asocia lo público (diversidad de actores) y lo político (estrategia de puesta en común entre los actores) (Alguacil, 2008:204). Sin embargo, en lo que respecta al envejecimiento y la vejez, tal participación alcanza ribetes no dimensionados del todo, ya que la participación no respondería solamente a la necesidad de que los envejecidos se organicen en pos de objetivos sociales o políticos comunes, sino también de que sean ellos mismos promotores y cuidadores de su salud. En efecto, se establece un ideal en donde la vejez participativa y asociativa genera instancias de colaboración enclaustrada en base a un criterio de edad, generándose, además, un principio de auto-responsabilidad y auto-cuidado que ha caracterizado la lógica del Estado para la gestión y el cálculo de grupos minoritarios.

Verónica Schild (2000) señala que el Estado neoliberal chileno, por medio del despliegue de mecanismos, prácticas y discursos que buscan empoderar a la población más vulnerable, intenta hacerlos responsables de su propia pobreza y exclusión. Por su parte, Julia Paley (2001) sostiene que los mecanismos de poder que operan durante el período democrático a través de la participación y de la construcción de sociedad civil representan un conjunto de prácticas y representaciones que canalizan la energía de las organizaciones sociales en un sentido funcional al nuevo modelo neoliberal de libre mercado [demostrando que] la idea de democracia participativa fue usada de manera estratégica por los gobiernos de la Concertación para limitar la movilización social y orientar el rol de la sociedad civil en el sistema político de la post-dictadura (citada en Boccara, 2008:193).

La necesidad de mantener a la población lo más activa y participativa posible, apunta en el caso de la vejez, que dicho segmento etáreo se mantenga de manera autónoma el mayor tiempo posible. Este es el principal desafío declarado por las agencias internacionales y por los diversos Estados, pues el coste de su mantención es el principal riesgo identificado hasta el momento. El Fondo Monetario Internacional (FMI), en este sentido, ha declarado que dicho riesgo, el riesgo de que la gente viva más de lo espera- 
do, afectará a las economías a nivel mundial, pues al ser el envejecimiento de la población una problemática subestimada en cuanto a su magnitud () disparará el coste previsto en decenas de billones de dólares a escala global. Eso supone una amenaza para la sostenibilidad de las finanzas públicas (Pozzi, 2012:1).

Como vemos hasta este punto, la complejidad del envejecimiento supera con creces las dimensiones hasta ahora abordadas por algunos estudios en ciencias sociales, pues la instrumentalización de la vida, en este caso, de la vida del viejo, tiene un claro objetivo: la búsqueda y mantención del equilibrio social producto de la amenaza cada vez más creciente de la vejez. La implementación de sutiles técnicas de gobierno, como hemos advertido al inicio de este trabajo, arremete de manera complementaria sobre el cuerpo individual y el cuerpo social (la población), sustentada en emplazamientos funcionales provenientes de instituciones estatales y académicas que apuntan a producir, en palabras de Foucault (2000), cuerpos dóciles: sumisión y utilización del cuerpo, que lo une al cuerpo analizable, al cuerpo manipulable Es dócil un cuerpo que puede ser sometido, que puede ser utilizado, que puede ser transformado y perfeccionado (Foucault, 2000:140). Así, proponemos el concepto de gerontogubernamentalidad para la observación y análisis de las nuevas artes de gobierno asociadas a la edad y su segmentación arbitraria y administrativa. Estas nuevas formas de gobierno se materializan en la diseminación, siempre en positivo, de discursos, prácticas de autocuidado y estilos de vida funcionales. En este sentido, parafraseando a Menéndez (1998), colocar en el estilo de vida del sujeto la responsabilidad de las formas de vida que lleva, es una variante de la culpabilización de la víctima. Estas lógicas contribuyen a formar subjetividades, a acrecentar la vida, a inducir a los usuarios a adoptar una buena conducta () a despolitizar los problemas de salud desvinculándolos de la estructura socioeconómica y a incitar a los individuos a que se gobiernen por sí mismos de la mejor manera posible. (Boccara, 2007:201). Ahora bien, en estos mecanismos, en estas lógicas asociadas al manejo de la vida, intervienen una serie de agentes que validan y reproducen dichas lógicas. Así, ciencia, técnica y política (binomio saber/poder), se clausuran para su reproducción y autovalidación.

\section{Discusión}

Hemos mostrado cómo la preocupación de la vejez, su gestión y su definición, han estado marcadas desde la década del 70 por una serie de agentes que han cimentado, producto de las tendencias demográficas, políticas que han buscado implementar lineamientos de acción en torno a este segmento de la población. Pretendemos, en este sentido, problematizar las abstracciones que hablan de envejecimiento y vejez como un fenómeno sincrónico y apolítico; como un fenómeno poblacional que emergió y en donde las instituciones de manera reactiva comienzan a operar. Desde un marco interpretativo biopolítico, consideramos al envejecimiento y la vejez como un fenómeno sobre el cual se generan cálculos con el objeto de 
disminuir los impactos negativos sobre la estabilidad social y económica de un país: paradojalmente, así fue primero con el baby boom, ahora con la vejez.

Atendiendo a la situación local, es posible observar el devenir del envejecimiento en la historia social mediante la gestión de la población que, siguiendo el camino trazado por la biopolítica occidental (Illanes, 2007), ha operado mediante la disminución de la mortalidad y el control familiar. Externalizando aspectos que tradicionalmente se mantenían al interior de la familia, como las conductas reproductivas o el cuidado de los envejecidos, comienzan a difuminar los límites entre lo privado y lo público. Lógicas similares y quizás más sutiles, como se expuso en las páginas precedentes, se aplican en el tratamiento de la vejez hoy por instituciones del Estado, y por cierto, por algunas instituciones académicas que reproducen, en pos de la optimización de la población envejecida, nociones y conceptos que se materializan en prácticas que promueven una vejez activa en centros gerontológicos, espacios recreativos o de formación como los propuestos por las Universidades para la Tercera Edad.

Foucault (1993) señala que el poder no sólo debe pensarse en la figura del Estado, sino en las redes, en las dinámicas microsociales, en el operar de las instituciones destinadas para estas funciones; también en los diversos flujos que, en el caso específico de la vejez, obedecen a lógicas transnacionales que se materializan bajo la noción de proyectos (en nuestro caso, del viejo-proyecto) y en fondos destinados para instalación de programas de investigación y académicos que buscan, por un parte, la producción de conocimiento local, y por otra, instalar y reproducir preceptos que modulan formas de envejecimiento exitoso y auto-responsables. Como hemos visto, la validez de estas lógicas ha radicado, y en la actualidad no está lejos de aquello, en los saberes que los expertos han producido bajo la figura de la salud pública.

Así, la salud pública es fundamental para la comprensión del gobierno de la vida, pues es la que intenta preservar la vida y promover nociones tales como la de salud colectiva dentro de los diversos dispositivos públicos creados para la gestión y control de la población, en especial, cuando una realidad epidemiológica como la del envejecimiento comienza a ser tan manifiesta. Se trata, entonces, de un dominio de actividad que da lugar a una especialización profesional e institucional: se forman médicos inspectores de salud pública. Es en fin, un campo disciplinario, con sus saberes, sus reglas, sus manuales, sus revistas, sus sociedades: departamentos o institutos de salud pública (Fassin, 2008:5). La salud pública no sólo tiene características objetivas, en este caso graficadas en el sumisión cuerpo individual y social a criterios de salud normativos, sino que también con cuestiones ideológicas en consecuencia a un proyecto antropológico que se fundamenta en las formas de nombrar y definir el mundo circundante y en las formas de considerar las variables culturales que, en la mayoría de los casos, son siempre analizadas en el lado de los destinatarios como elementos que deben ser sometidos a traducción en términos de comportamientos 
patogénicos o protectores que son ingresados al cálculo de probabilidades de riesgos a los cuales se exponen los individuos () (ibid.:17) circunscritos en el reconocimiento de un bien común o valores comunes.

Se desprende acá otra dimensión a considerar de manera un tanto más detallada, la cuestión de la construcción o traducción del hecho biológico a un hecho social y cómo, a partir de esto, la producción y circulación de conceptos fundamentales permiten fijar un fenómeno en el marco de la salud pública. Para graficar esta dimensión, nos apoyamos en un ejemplo que ofrece Fassin en su artículo La Patetización del Mundo (1999), en donde muestra cómo los pobres se convierten en excluidos: en la última década, en Francia, la palabra desigualdades desapareció prácticamente del léxico político y científico; fue reemplazada por el término exclusión () este cambio de vocabulario no es anecdótico. Por el contrario, es revelador de una nueva representación del espacio social, de una nueva topografía simbólica de la sociedad (Fassin, 1999:34). Este fenómeno no se encuentra lejos de la situación del envejecimiento y la vejez, puesto que nominaciones como ancianidad, tercera o cuarta edad, vejez activa, productiva, entre otras, corresponden a descripciones normativas y criterios políticos sustentados en un proyecto antropológico que, además, sedimenta el ejercicio del poder sobre la vida.

Creemos que al pensar el envejecimiento y vejez como construcciones por parte de las disciplinas que han monopolizado el saber en torno a los cuerpos envejecidos, es decir, las ciencias biológicas y médicas, evidenciaremos precisamente las lógicas motivadoras subyacentes en el quehacer académico y científico en torno a la vejez; y cómo éstas, en los diversos sistemas de inscripciones utilizados en el campo científico (clases, artículos, investigaciones), inciden y hacen efectiva tal construcción en el campo político, lo público o lo que quedaría, quizá, de lo privado. 


\title{
Notas
}

${ }^{1}$ Parte del contenido de este artículo corresponde a la revisión bibliográfica de la tesis del autor para optar al grado académico de Magíster en Antropología en las Universidades Católica del Norte y Tarapacá, Chile.

Una versión preliminar de este texto fue presentada en el Seminario Física y Química: Diálogos fronterizos en torno al cuerpo, organizado por el Departamento de Sociología I (Cambio Social) en la Universidad Complutense de Madrid, España, en el mes de febrero del año 2012. Dicha presentación fue realizada en el marco de una estancia de investigación financiada por la Beca para doctorandos en tesis del Programa MECESUP2. Agradezco a la evaluadora o evaluador anónima/o por las críticas realizadas a este texto; todo error o inconsistencia en el artículo es de exclusiva responsabilidad del autor.

${ }^{2}$ Un interesante texto que muestra los cambios ocurridos en la familia chilena como resultado del proceso de modernización, es el trabajo de Gutiérrez y Osorio (2008), cuyo énfasis está puesto en lo que ellos denominan el proceso de acondicionamiento de dos generaciones (siglo XX) y las relaciones familiares (parejas y padres/hijos) con un énfasis en el rol del Estado en estos temas.

${ }^{3}$ Las actuales lecturas de la biopolítica analizan temas como: educación, economía, el medioambiente e inclusive, respecto a los avances de la biotecnología aplicada a las poblaciones en el marco de la modernidad biológica propuesta por Foucault (2002a).

\begin{abstract}
${ }^{4}$ Se hace referencia, por una parte, al rol del Estado en las temáticas de salubridad, señalando: Es deber del Estado velar por la salud pública y el bienestar higiénico del país. Deberá destinarse cada año una cantidad de dinero suficiente para mantener un servicio nacional de salubridad () (Capítulo III, Garantías Constitucionales, Inciso $14^{\circ}$ ), y por otro, el rol del municipio en estas materias en el Capítulo IX, artículo 105, Inciso $1^{\circ}$.
\end{abstract}

${ }^{5}$ Para una revisión más detallada de estas materias, ver los trabajos de Salinas (1983), Illanes (2007), Zárate (2008) y Rojas (2009).

${ }^{6}$ Benjamín Viel Vicuña (1913 - 1998). Fue profesor en la Escuela de Medicina de la Universidad de Chile donde tenía a cargo la cátedra Medicina Social y Preventiva. Considerado pionero en las estrategias de planificación familiar a nivel nacional.

${ }^{7}$ Eso sí, debemos considerar que la pretensión de explicar el cambio demográfico exclusivamente por las políticas de planificación familiar resultaría insuficiente. Existe una dimensión de orden sociológico vinculada a la autodeterminación del cuerpo de las mujeres que sería el campo propicio para este tipo de iniciativas de control familiar. Todo este proceso se enmarcaría en los movimientos sociales que, desde 1913, buscaban cambiar el rol de las mujeres en la sociedad chilena y sus derechos ciudadanos, siendo el derecho al voto femenino alcanzado en 1949, el hecho que marcaría este proceso.

${ }^{8}$ En términos generales, se han descrito en la literatura cuatro etapas vinculadas a las transiciones demográficas y epidemiológicas experimentadas en los diversos países del globo. La primera etapa, transición incipiente, se caracteriza por altas tasas de mortalidad y fecundidad. La transición moderada se caracteriza por una alta tasa de fecundidad y la mortalidad comienza a descender. La transición plena está caracterizada por las tasas de fecundidad y mortalidad que se encuentran en disminución. Finalmente, la etapa de transición avanzada o muy avanzada, se caracteriza por la franca caída de las 
tasas de fecundidad y natalidad.

${ }^{9}$ Interesantes son los trabajos de Terrón (2004) y Viveros (2007), quienes enuncian los diversos eventos producidos por las agencias internacionales desde 1977. A nuestro juicio, el hecho de mayor significancia en términos analíticos es la relación que hace la ONU, en 1995, del informe sobre los derechos de las personas mayores y el Pacto Internacional de Derechos Económicos, Sociales y Culturales, U.N, Doc, E/C.12/1995/16/Rev.1 de 1995, vinculándose de esta manera la vejez a los Derechos Humanos. Sobre esta dimensión cabe señalar lo siguiente: desde este punto se comienza a comprender la situación social de la población envejecida enmarcada en una lógica de derechos universales, no obstante, al enlazar a la población envejecida a una serie de derechos específicos como los económicos, sociales y culturales, se construye la imagen de una población en desprotección frente a factores externos que hacen peligrar el ejercicio de estos derechos.

${ }^{10}$ Desde una perspectiva más general a partir del siglo XX, la preocupación del estudio de la dinámica poblacional ha despertado gran interés más allá de las simples cuentas censales para calcular tributaciones, medir potenciales para defender la patria, llenar necesidades de ocupar y poblar territorios vacíos (Ham, 2003:31), ya que la consideración de temas relacionados a la educación, la salud, trabajo, pobreza, etc., ha puesto en alerta, en particular, a los países en vías de desarrollo a pensar también las cuestiones de orden demográfico en conjunto con las políticas de desarrollo. En este sentido, se han realizado seis conferencias internacionales sobre Población y Desarrollo organizadas por las Naciones Unidades: Roma (31 de agosto al 10 de septiembre de 1954), Belgrado (organizada en conjunto con la International Union for the Scientific Study of Population IUSSP, 30 de agosto al 10 de septiembre de 1965), Bucarest (19 al 30 de agosto de 1974), Ciudad de México (6 al 14 de agosto de 1984), El Cairo (5 al 13 de septiembre de 1994) y Nueva York (30 de junio al 2 de julio de 1999).

${ }^{11}$ Lo público difumina los límites de lo privado, pues monopolizar el término público en vez de lo colectivo, por ejemplo, sitúa la problemática inmediatamente en el campo de la política, en donde nociones como integridad de los cuerpos y la preservación de su existencia, implicarían en lo formal responsabilidad de la propia sociedad y de los sujetos, así como de aquellas instituciones estatales u otras vinculadas a la salud.

${ }^{12}$ Producto de los avances en medicina, el deterioro en la vejez es cada vez más aplazado, por ello, genera aún mayor complejidad el poder fijar conceptos como el de cuarta edad.

${ }^{13}$ Para mayores detalles del surgimiento de estas instituciones, sugerimos ver Ríos (1999) y Morales (2010).

${ }^{14}$ Por otro lado, de manera paralela, se crea el sistema de salud privado ISAPRES (Instituciones de Salud Previsional).

${ }^{15}$ CONAPRAN, que también se perfiló como una institución de carácter asistencial, su principal función en aquella época era la de agrupar a la población mayor en clubes con claros objetivos recreativos. Posterior el régimen militar, esta institución ha mantenido su funcionamiento y el carácter de beneficencia.

${ }^{16}$ Los clubes de tercera edad, establecidos en dependencias comunales y de la iglesia, mayoritariamente, constituyen entidades comunitarias en las cuales las personas mayores se organizaban en un número rara vez superior a treinta personas () así, expresión afectiva y 
esparcimiento constituyen los móviles centrales de la participación del adulto mayor en la mayoría de estas organizaciones. (Ríos, 1999:37).

${ }^{17}$ Por otro lado, es importante considerar otras instituciones de carácter benéfico que han tenido un rol importante en la gestión de la población envejecida: la Iglesia Católica, el Ejército de Salvación, Caritas Chile, el Club de Leones y Hogar de Cristo, son las más representativas en este sentido (Gutiérrez, 2006), y organizaciones de la sociedad civil como los clubes del adulto mayor.

${ }^{18} \mathrm{Al}$ igual como en los países del viejo continente, el sistema de pensiones es uno de los ejes de mayor tensión y reformulación. En este sentido, la Reforma Previsional aprobada en enero de 2008, que crea un Sistema de Pensiones Solidarias que beneficia a quienes () no se han incorporado a un sistema previsional o no tienen derecho a pensión en algún régimen previsional o que encontrándose afiliados a algún sistema, habiendo cotizado y cumpliendo con los requisitos de elegibilidad, no han logrado reunir los fondos suficientes para financiar una pensión digna. (Yañez, 2010:28), busca sortear esta tensión.

${ }^{19} \mathrm{Si}$ bien el envejecimiento activo, exitoso, productivo y otras tantas tautologías difieren conceptualmente desde el saber experto, nosotros proponemos homologar dichas categorías, pues apuntan a un fin común: el bienestar de la población en miras de la reducción del coste que significa a los Estados la mantención de la población envejecida.

${ }^{20}$ En total, corresponden a 253 consejeros distribuidos en cada región del país.

${ }^{21}$ Entrevista realizada en las dependencias del SENAMA en el mes de junio del año 2010.

${ }^{22}$ En este sentido, algunos avances respecto del concepto de biopolítica nos sirven para explicar de mejor manera nuestro análisis. Para Esposito (2005), la protección de la vida se encuentra mediada por una reproducción controlada del mal de que se debe proteger. Protección de la vida por medio de la negación de la misma a pequeña escala. Inmunización es el concepto propuesto: () mediante la protección inmunitaria la vida combate lo que niega, pero según una ley que no es la de contraposición frontal, sino la del rodeo y la neutralización. El mal debe enfrentarse, pero sin alejarlo de los propios confines. Al contrario, incluyéndolo dentro de estos confines. La figura dialéctica que de este modo se bosquejó es la de una inclusión excluyente o de una exclusión mediante la inclusión (Esposito, 2005:17-18). 


\section{Bibliografía}

Agamben, G. (2006), Homo Sacer. El poder soberano y la nuda vida. Editorial Pre-Textos. Valencia.

Alguacil, J. (2008), “Espacio público y espacio político. La ciudad como el lugar para las estrategias de participación”. En Polis. Revista Latinoamericana. Vol.7, N²0, 199-223.

Boccara, G. (2007), “Etnogubernamentalidad. La formación del campo de la salud intercultural en Chile”. En Estudios Atacameños. 39, N², 187207.

Boccara, G. y Bolados, P. (2008), “¿Dominar a través de la participación? Neoindigenismo en el Chile de la postdictadura”. En Memoria Americana. 16 (2), 167-196.

Bolados, P. (2009), “¿Participación o pacificación social? La lógica neoliberal en el campo de la salud intercultural en Chile: El caso Atacameño”. En Estudios Atacameños. N³8, 93-106.

Bourdieu, P. (2005), "El ministerio del ministerio. De las voluntades particulares a la 'voluntad general' ”. En Wacquant, L. (Coord.), El Ministerio del ministerio. Pierre Bourdieu y la política democrática. Editorial Gedisa. Barcelona.

Ídem (2007), El sentido práctico. Siglo XXI Editores. Buenos Aires.

Castro, E. (2008), “Biopolítica: de la soberanía al gobierno”. En Revista Latinoamericana de Filosofía. Vol. XXXIV, N²:187-205.

Ídem (2010), “¿Qué es la política para la biopolítica?”. En Cassigoli, I. y Sobarzo, M. (Eds.), Biopolíticas del sur. (pp.21-33). Editorial ARCIS, Santiago de Chile.

Criado, E. (1998), Producir la juventud. Ediciones ISTMO. Madrid.

Esposito, R. (2005), Inmunitas. Protección y negación de la vida. Amorrortu Editores. Buenos Aires.

Ídem (2006), Bíos. Biopolítica y filosofía. Amorrortu Editores. Buenos Aires.

EXPANSIVA. (2008), Construyendo Políticas Públicas para una Sociedad que Envejece. Autor, Universidad de Chile.

Fassin, D. (1999), “La patetización del mundo. Ensayo de antropología política del sufrimiento”. En Viveros, M. y Garay, G. (Comp.), Cuerpo, Diferencias y Desigualdades. (pp.31-41). Santafé de Bogotá. 
Ídem (2004), "Entre las políticas de lo viviente y las políticas de la vida”. En Revista Colombiana de Antropología. 40:283-318.

Ídem (2008), El hacer de la salud pública. Recuperado el 23 de marzo 2010, de http://www.librosintinta.in/biblioteca/ver-pdf/www.equidad.cl/ biblioteca/formador-de-formadores/modulo-1/El\%20hacer\%20de\% 20la\%20salud\%20p\%C3\%BAblica\%20Didier\%20Fassin.pdf.htx

Foucault, M. (1993), Microfísica del poder, Editorial La Piqueta, Madrid. Ídem (1995), El Nacimiento de la clínica. Una arqueología de la mirada médica. Siglo XXI Editores. España.

Ídem (2000), Vigilar y castigar. Siglo XXI Editores. Madrid.

Ídem (2002a), Historia de la sexualidad. 1-La voluntad de saber. México. Siglo XXI Editores. España.

Ídem (2002b), Defender la sociedad. Fondo de Cultura Económica. Buenos Aires.

Ídem (2007), Seguridad, territorio, población. Fondo de Cultura Económica. Buenos Aires.

Ídem. (2008), El nacimiento de la biopolítica. Fondo de Cultura Económica. Buenos Aires.

Gutiérrez, E. y Osorio, P. (2008), “Modernización y transformaciones de las familias como procesos del condicionamiento social de dos generaciones”. En Revista Última Década. 29: 103-35.

Gutiérrez, E. y Ríos, P. (2006), "Envejecimiento y campo de la edad: elementos sobre la pertinencia del conocimiento gerontológico". En Revista Última Década 25:11-41.

Gutiérrez, E., Osorio, P., Ríos, P. y Wilson, C. (2006), Adultez mayor. contexto + subtexto. Antología de textos gerontológicos. Hacia la construcción de una nueva agenda. Observatorio Social Envejecimiento y Vejez, Universidad de Chile.

Ham, R. (2003), El envejecimiento en México: El siguiente reto de la transición demográfica. El Colegio de la Frontera Norte, México.

Illanes, M. (2007), Cuerpo y sangre de la política. La construcción histórica de las visitadoras sociales (1887 1940). LOM Ediciones. Santiago de Chile.

Law, J. (1998), "Del poder y sus tácticas. Un enfoque desde la sociología de la ciencia”. En Domènech, M. y Tirado, F.J. (Comp.), Sociología Simé- 
trica. Ensayos sobre Ciencia, Tecnología y Sociedad. (pp.63-107). GEDISA Editorial. Barcelona.

Menéndez, E. (1998), “Estilos de vida, riesgos y construcción social”. Conceptos similares y significados diferentes. En Estudios sociológicos. Año XVI, 46: 37-67.

Morales, M. (2001), "Los adultos mayores chilenos en el siglo XXI: Un enfoque politológico”. En Acta Bioethica. Año VII, N¹, 71-95.

Morris, P. (2008), Políticas para el adulto mayor en Chile: Institucionalización y desafíos políticos y técnicos. Recuperado el 23 de marzo 2009, de http://www.bcn.cl/carpeta_temas_profundidad/ temas_profundidad.2008-04-30.0122799562/documentos_pdf.2008-0430.1359051441/archivos_pdf.2008-04-30.2862146129/

Osorio, P. (2006a), “Exclusión generacional: La tercera edad”. En Revista MAD, Número Especial: Nuevas Exclusiones en la Complejidad Social Contemporánea. 1:177-186.

Ídem (2006b), La longevidad: Más allá de la biología. Aspectos socioculturales. Recuperado el 23 de marzo 2008, de http:// www.identidadcolectiva.es/pdf/22.pdf

Ídem (2006c), Abordaje Antropológico del envejecimiento y el alargamiento de la vida. Recuperado el 03 de marzo 2009, de http://www.facso.uchile.cl/ observa/12_oct_alargamiento.pdf

Palma, I. (2006), Transformaciones de la fecundidad en la sociedad chilena. Recuperado el 07 de enero 2009, de http://www.pasa.cl/wp-content/ uploads/2011/08/Transformaciones_de_la_Fecundidad_en_la_ Sociedad_Chilena_Palma_Irma.doc

Pieper, J. (2008), “Salvar vidas y gestar la modernidad: Médicos, mujeres y programas de planificación familiar en Chile”. En Zárate, M.S. (Comp.), Por la Salud del Cuerpo. Historia y Políticas Sanitarias en Chile. (pp. 189 - 228). Universidad Alberto Hurtado. Santiago de Chile.

Pozi, S. (2012, 11 de abril), "El FMI pide bajar pensiones por el riesgo de que la gente viva más de lo esperado”. El País. Recuperado el 11 de abril de 2012, de http://economia.elpais.com/economia/2012/04/11/actualidad/ 1334133453_457282.html

Rojas, C. (2009), "Lo global y lo local en los inicios de la planificación familiar en Chile”. En Estudios Avanzados, USACH. 11:7-27.

Salinas, R. (1983), “Salud, ideología y desarrollo social en Chile 1830 1950”. En Cuadernos de Historia Universidad de Chile 3: 99 -126. 
SENAMA. (2009), Las personas mayores en Chile. Situación, avances y desafíos del envejecimiento y vejez. Santiago, Chile.

Senellart, M. (2007), “Situación de los cursos”. En Foucault, M. Seguridad, territorio, población. (pp.417-453). Fondo de Cultura Económica. Buenos Aires.

Schild, V. (2000), “Neo-liberalisms New Gendered Market Citizens: The Civilizing Dimension of Social Programs in Chile”. En Citizenship Studies, Volume 4, Issue 3:275-305.

Terrón, D. (2004), “El régimen jurídico de las personas mayores. Especial referencia a la situación de Castilla y León”. En Alonso, M., Martínez, E. y Reguero, J. (Coords.), Protección Jurídica de los Mayores. La LeyActualidad. Madrid, España.

Villalón, G. y Vera, S. (2012), “Panorama demográfico en Chile contemporáneo: Desafíos para la sociedad del Siglo XXI”. En Revista Anales de la Universidad de Chile, Séptima serie 3: 35-63.

Viveros, A. (2007), “Acerca del envejecimiento y la vejez: Notas para dimensionar la acción de la sociedad sobre el tema”. En Revista de Ciencias Sociales 18: 125-141.

Yañez, S. (2010), La dimensión de género en la reforma previsional chilena (Ley $N^{\circ} 20.255$ de reforma de pensiones). Naciones Unidas, Santiago, Chile.

Zárate, M. (Comp.) (2008), Por la salud del cuerpo. Historia y políticas sanitarias en Chile. Ediciones Universidad Alberto Hurtado. Santiago, Chile.

Recibido: 11.06.2012

Aceptado: 10.04.2013 